\title{
Evidence for the Placement of the Gram-Negative Catonella morbi (Moore and Moore) and Johnsonella ignava (Moore and Moore) within the Clostridium Subphylum of the Gram-Positive Bacteria on the Basis of $16 \mathrm{~S}$ rRNA Sequences
}

\author{
ANNE WILLEMS* AND MATTHEW D. COLLINS \\ Department of Microbial Physiology, Institute of Food Research, Reading Laboratory, \\ Reading RG6 6BZ, United Kingdom
}

\begin{abstract}
Comparative 16S rRNA analysis was used to determine the phylogenetic positions of Catonella morbi and Johnsonella ignava, which are members of two monospecific genera of gram-negative anaerobic bacilli isolated from human gingival crevices. Both of these genera were found to belong to cluster XIVa (M. D. Collins, P. A. Lawson, A. Willems, J. J. Cordoba, J. Fernandez-Garayzabal, P. Garcia, J. Cai, H. Hippe, and J. A. E. Farrow, Int. J. Syst. Bacteriol. 44:812-826, 1994) of the Clostridium subphylum of gram-positive bacteria. Within this cluster, which contains several Clostridium, Coprococcus, Eubacterium, and Ruminococcus species, $C$. morbi and J. ignava formed two distinct lines that were separate from all other taxa. Our findings support the separate generic status of the genera Catonella and Johnsonella and show that these genera do not belong to the family Bacteroidaceae but instead belong to the gram-positive Clostridium subphylum.
\end{abstract}

Recently, Moore and Moore (9) described five new genera (the genera Catonella, Dialister, Hallella, Johnsonella, and Oribaculum) within the family Bacteroidaceae for groups of anaerobic, gram-negative bacilli that occur in human gingival crevices and periodontal pockets. In previous studies $16 \mathrm{~S}$ rRNA gene sequence analysis was used to determine the phylogenetic positions of three of these new genera. Dialister pneumosintes (formerly Bacteroides pneumosintes) was found to belong to the Sporomusa subbranch of the Clostridium subphylum of gram-positive bacteria (10). In contrast, Hallella seregens and Oribaculum catoniae grouped within the Bacteroidaceae but formed separate lineages within the genera Prevotella and Porphyromonas, respectively $(11,12)$. In this paper, we describe the phylogenetic positions of members of the remaining two genera, Catonella morbi and Johnsonella ignava.

Catonella morbi, formerly known as "Bacteroides D42," was described for a group of gram-negative saccharolytic bacilli associated with periodontitis. Catonella morbi strains have a $\mathrm{G}+\mathrm{C}$ content of $34 \mathrm{~mol} \%$ (9) and can be distinguished from members of other genera of gram-negative anaerobes with similar $\mathrm{G}+\mathrm{C}$ contents by the fact that in peptone-yeast extractglucose (PYG) broth they produce predominantly acetic acid and moderate amounts of formic and lactic acids (9). J. ignava was described for a group of gram-negative, nonfermentative, nonproteolytic bacilli that were previously referred to as " $\mathrm{Bac}$ teroides D19" (9) and are associated with gingivitis and periodontitis. J. ignava has a $\mathrm{G}+\mathrm{C}$ content of $32 \mathrm{~mol} \%$ and produces moderate amounts of acetate and isovalerate and trace amounts of lactate, succinate, and isobutyrate in PYG broth (9). In this study we determined the 16S rRNA gene sequences of the type strains of Catonella morbi and J. ignava in order to investigate the phylogenetic distinctiveness and higher affiliations of these taxa.

Lyophilized cells of Catonella morbi ATCC $51271^{\mathrm{T}}(\mathrm{T}=$ type strain) and $J$. ignava ATCC $51276^{\mathrm{T}}$ were obtained from

* Corresponding author. Mailing address: Department of Microbial Physiology, Institute of Food Research, Reading Laboratory, Earley Gate, Whiteknights Road, Reading RG6 6BZ, United Kingdom. the American Type Culture Collection, Rockville, Md. DNA was extracted directly from freeze-dried cells, resuspended in $500 \mu \mathrm{l}$ of TES buffer $(0.05 \mathrm{M}$ Tris- $\mathrm{HCl}, 0.005 \mathrm{M}$ EDTA, 0.05 $\mathrm{M} \mathrm{NaCl}$; $\mathrm{pH} \mathrm{8.0),} \mathrm{as} \mathrm{described} \mathrm{by} \mathrm{Lawson} \mathrm{et} \mathrm{al.} \mathrm{(8).} \mathrm{A} \mathrm{PCR}$ fragment containing the almost-complete 16S rRNA gene (positions 30 to 1521 ; Escherichia coli numbering) was generated by using primers for highly conserved sequences at the $3^{\prime}$ and $5^{\prime}$ ends of the gene. PCR products were cleaned by using a Prep-A-Gene kit (Bio-Rad, Hercules, Calif.) as recommended by the manufacturer and were sequenced directly by using a Taq DyeDeoxy terminator cycle sequencing kit (Applied Biosystems, Foster City, Calif.) and an Applied Biosystems model 373A automatic DNA sequencer.

A search of the EMBL and GenBank data libraries was performed by using the FASTA program of the Genetics Computer Group sequence analysis package (3) to determine the sequences most similar to the newly determined sequences. On the basis of the information obtained a set of sequences of reference organisms was retrieved from the EMBL, GenBank, Ribosomal Database and Project databases for further analysis. The sequences were aligned by using the PILEUP and PRETTY programs (3), and the multiple alignment was then corrected manually. Approximately 100 nucleotides at the 5' end of the molecule were omitted from the analysis because of alignment ambiguities; this resulted in a continuous stretch of 1,306 nucleotides being used for each strain in the analysis. Using the PHYLIP package (5), we constructed a distance matrix with the program DNADIST (by using the Kimura-2 parameter), and a phylogenetic tree was produced by using the neighbor-joining method with the program NEIGHBOR. The stability of the groups obtained was verified by the bootstrapping method ( 500 bootstrap replications) by using the programs SEQBOOT, DNADIST, NEIGHBOR, and CONSENSE of the PHYLIP package. For comparison, a pairwise similarity matrix was constructed by repeatedly using the GAP program of the Genetics Computer Group package; the similarity values were converted to nucleotide substitution values $\left(K_{\text {nuc }}\right.$ values [7]), and a phylogenetic tree was drawn by using the NEIGHBOR program. A parsimony analysis was per- 
formed with the multiple sequence alignment by using the DNAPARS program in the PHYLIP package.

The 16S rRNA sequences which we determined for $\mathrm{Ca}$ tonella morbi ATCC $51271^{\mathrm{T}}$ and J. ignava ATCC $51276^{\mathrm{T}}$ consisted of 1,467 and 1,478 nucleotides, respectively. Preliminary database searches revealed that both of these species were only remotely related to members of the gram-negative family $\mathrm{Bac}$ teroidaceae (levels of sequence similarity, approximately 71 to $74 \%$ ) and were more closely affiliated with the Clostridium subphylum of gram-positive bacteria. To determine the positions of Catonella morbi and $J$. ignava within this subphylum, we first performed distance matrix-based phylogenetic analyses (by using both multiple and pairwise sequence alignments) with 75 strains representing the 19 clostridial clusters described by Collins et al. (2), as well as the Bacteroidaceae, the Proteobacteria, the Lactobacillaceae, the Bacillaceae, the Mycoplasmatales, and the Actinomycetales. These analyses (data not shown) revealed that both Catonella morbi and $J$. ignava belong to cluster XIVa of the Clostridium subphylum as defined by Collins et al. (2). Cluster XIVa contains several Clostridium, Coprococcus, Eubacterium, and Ruminococcus species, as well as Acetitomaculum ruminis. To assess the relationships of $\mathrm{Ca}$ tonella morbi and $J$. ignava within cluster XIVa, we performed additional phylogenetic analyses in which we included all known species belonging to this cluster, as well as cluster $\mathrm{XIVb}$, and Clostridium butyricum (cluster I), the type species of the genus Clostridium, as an outgroup. Catonella morbi and $J$. ignava exhibited levels of sequence similarity of approximately 84 to 88 and 84 to $87 \%$, respectively, with other taxa belonging to cluster XIVa, and a level of sequence similarity of $84.6 \%$ with each other. The three approaches which we used for sequence analysis (distance matrix analysis based on both multiple and pairwise sequence alignments and parsimony analysis) resulted in very similar phylogenetic trees and clearly showed that within cluster XIVa Catonella morbi and J. ignava belong to distinct sublineages. For example, the phylogenetic tree obtained from a neighbor-joining analysis of a distance matrix calculated from the multiple sequence alignment is shown in Fig. 1. The low bootstrap values obtained for $\mathrm{Ca}$ tonella morbi and $J$. ignava indicate that there is considerable uncertainty concerning the precise positions of these organisms within cluster XIVa, although the lengths of the branches clearly support recognition of these taxa as members of separate genera (9). All groups with bootstrap values of $80 \%$ or more (Fig. 1) were also recovered in phylogenetic trees (data not shown) obtained by performing a distance matrix analysis after pairwise sequence alignment and by performing a parsimony analysis (three most parsimonious trees were obtained [data not shown]). Eubacterium saburreum was consistently recovered as the closest neighbor of $J$. ignava in all of the analyses performed. However, the observed level of sequence divergence (13\%) and the low bootstrap value (Fig. 1) indicate that this relationship is probably not particularly significant.

The finding that gram-negative genera group together with gram-positive genera is quite surprising, but not exceptional. So far, most gram-negative organisms that are phylogenetically related to the clostridia belong to the Sporomusa subbranch. To our knowledge, the only other example of a gram-negative species that belongs to one of the other clostridial clusters is Tissierella praeacuta, which belongs to cluster XII and is closely related genealogically to Clostridium hastiforme (4). In this context it is worth noting that although some organisms (e.g., Butyrivibrio species) are considered gram-negative when traditional staining methods are used, they have been shown by ultrastructural electron microscopy studies to possess unusually thin but gram-positive cell walls $(1,6)$.

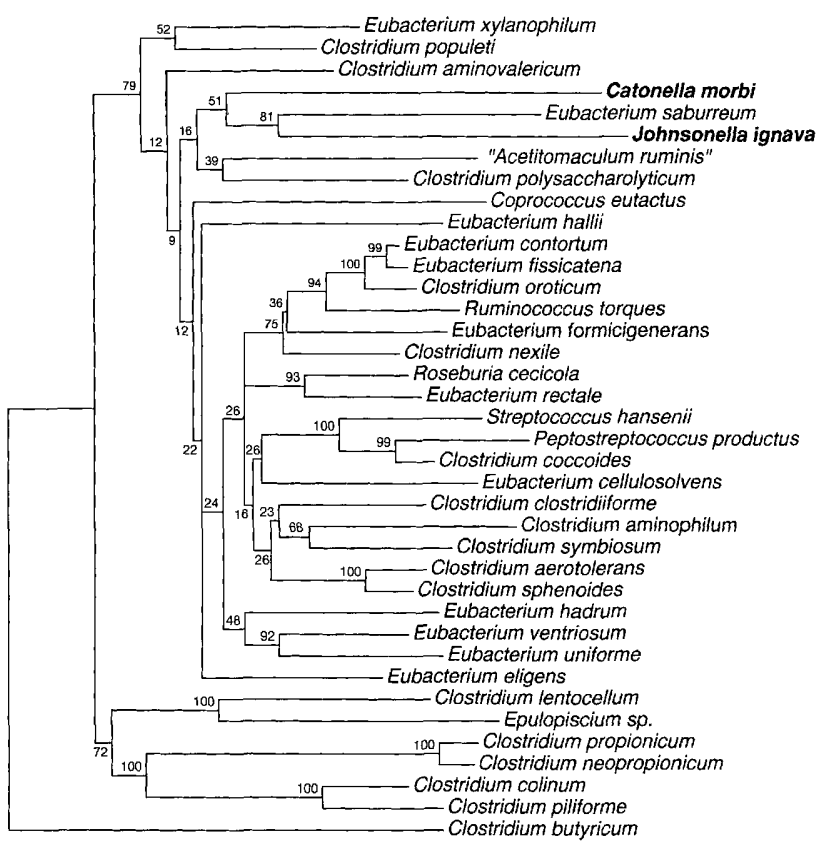

FIG. 1. Unrooted phylogenetic tree obtained by a neighbor-joining analysis of a distance matrix calculated from a multiple sequence alignment, showing the positions of Catonella morbi and $J$. ignava within the Clostridium subphylum of gram-positive bacteria. Bootstrap values (expressed as percentages of 500 replications) are indicated at the branch points; values of $90 \%$ or more were considered significant.

The 16S rRNA gene sequences of Catonella morbi ATCC $51271^{\mathrm{T}}$ and $J$. ignava ATCC $51276^{\mathrm{T}}$ have been deposited in the EMBL data library under accession numbers X87151 and $\mathrm{X} 87152$, respectively.

We gratefully acknowledge the support of Commission of the European Communities grant Bio-2-CT93-0119.

\section{REFERENCES}

1. Cheng, K.-J., and J. W. Costerton. 1977. Ultrastructure of Butyrivibrio fibrisolvens: a gram-positive bacterium? J. Bacteriol. 129:1506-1512

2. Collins, M. D., P. A. Lawson, A. Willems, J. J. Cordoba, J. FernandezGarayzabal, P. Garcia, J. Cai, H. Hippe, and J. A. E. Farrow. 1994. The phylogeny of the genus Clostridium: proposal of five new genera and eleven new species combinations. Int. J. Syst. Bacteriol. 44:812-826.

3. Devereux, J., P. Haeberli, and D. Smithies. 1984. A comprehensive set of sequence analysis programs for the VAX. Nucleic Acids Res. 12:387-395.

4. Farrow, J. A. E., P. A. Lawson, H. Hippe, U. Gauglitz, and M. D. Collins. 1995. Phylogenetic evidence that the gram-negative nonsporulating bacterium Tissierella (Bacteroides) praeacuta is a member of the Clostridium subphylum of the gram-positive bacteria and description of Tissierella creatinini sp. nov. Int. J. Syst. Bacteriol. 45:436-440.

5. Felsenstein, J. 1989. PHYLIP-phylogeny inference package (version 3.2). Cladistics 5:164-166.

6. Hespell, R. B., K. Kato, and J. W. Costerton. 1993. Characterization of the cell wall of Butyrivibrio species. Can. J. Microbiol. 39:912-921.

7. Hori, H., and S. Osawa. 1979. Evolutionary change in 5S rRNA secondary structure and phylogenetic tree of 54 5S rRNA species. Proc. Natl. Acad. Sci. USA 76:381-385.

8. Lawson, P. A., S. E. Gharbia, H. N. Shah, and D. R. Clark. 1989. Recognition of Fusobacterium nucleatum subgroups $\mathrm{Fn}-1, \mathrm{Fn}-2$ and $\mathrm{Fn}-3$ by ribosomal RNA gene restriction patterns. FEMS Microbiol. Lett. 65:41-46.

9. Moore, L. V. H., and W. E. C. Moore. 1994. Oribaculum catoniae gen. nov., sp. nov.; Catonella morbi gen. nov., sp. nov.; Hallella seregens gen. nov., sp. nov.; Johnsonella ignava gen. nov., sp. nov.; and Dialister pneumosintes comb. nov., nom. rev., anaerobic gram-negative bacilli from the human gingival crevice. Int. J. Syst. Bacteriol. 44:187-192.

10. Willems, A., and M. D. Collins. 1995. Phylogenetic placement of Dialister 
pneumosintes (formerly Bacteroides pneumosintes) within the Sporomusa subbranch of the Clostridium subphylum of the gram-positive bacteria. Int. J. Syst. Bacteriol. 45:403-405.

11. Willems, A., and M. D. Collins. 1995. Reclassification of Oribaculum catoniae (Moore and Moore 1994) as Porphyromonas catoniae comb. nov. and emendation of the genus Porphyromonas. Int. J. Syst. Bacteriol. 45:578-581.
12. Willems, A., and M. D. Collins. 1995. 16S rRNA gene similarities indicate that Hallella seregens (Moore and Moore) and Mitsuokella dentalis (Haapasalo et al.) are genealogically highly related and are members of the genus Prevotella (Shah and Collins): emended description of the genus Prevotella (Shah and Collins) and description of Prevotella dentalis comb. nov. Int. J. Syst. Bacteriol. 45:832-836. 